\title{
Kernel perfect and critical kernel imperfect digraphs structure
}

\author{
Hortensia Galeana-Sánchez ${ }^{1 \dagger}$ and Mucuy-Kak Guevara ${ }^{1 \ddagger}$
}

${ }^{1}$ Instituto de Matemáticas, Circuito Exterior, C.U. México 04510 D.F. México.

\begin{abstract}
A kernel $N$ of a digraph $D$ is an independent set of vertices of $D$ such that for every $w \in V(D)-N$ there exists an arc from $w$ to $N$. If every induced subdigraph of $D$ has a kernel, $D$ is said to be a kernel perfect digraph. Minimal non-kernel perfect digraph are called critical kernel imperfect digraph. If $F$ is a set of arcs of $D$, a semikernel modulo $F, S$ of $D$ is an independent set of vertices of $D$ such that for every $z \in V(D)-S$ for which there exists an $S z-\operatorname{arc}$ of $D-F$, there also exists an $z S-\operatorname{arc}$ in $D$. In this talk some structural results concerning critical kernel imperfect and sufficient conditions for a digraph to be a critical kernel imperfect digraph are presented.
\end{abstract}

Keywords: kernel, semikernel, semikernel modulo F, kernel perfect digraph, critical kernel imperfect digraph

Let $D$ be a digraph; $V(D)$ and $A(D)$ will denote the set of vertices and $\operatorname{arcs}$ of $D$ respectively. Let $S_{1}, S_{2}$ be subsets of $V(D)$. The arc $u_{1} u_{2}$ of $D$ will be called an $S_{1} S_{2}-\operatorname{arc}$ whenever $u_{1} \in S_{1}$ y $u_{2} \in S_{2}$. Let $H$ be a subdigraph of $D$. If $u v \in A(D)-A(H)$ then $u v$ is called a pseudodiagonal of $H . \Gamma^{+}(u)$, (resp. $\Gamma^{-}(u)$ ) is the exneighbourhood (resp. inneighbourhood) of $u$ in $D$.

A kernel $N$ of $D$ is an independent set of vertices such that for every $w \in V(D)-N$ there exists an arc from $w$ to a vertex in $N$. The concept of kernel was introduced by Von Neumann and Morgenstern (10) as an abstract generalization of their concept of solution for cooperative games. The problem of the existence of a kernel in a given digraph has been studied by several authors, since it is important in the context of Game Theory and Decision Theory, so the main question is: Which structural properties of a graph imply the existence of a kernel?

The classical results (1) are:

1. A symmetric digraph is kernel perfect;

2. A transitive digraph is kernel perfect, and all kernels have the same cardinality (König);

3. A digraph without cycles is kernel perfect, and its kernel is unique (von Neumann);

4. A graph without cycles of odd length is kernel perfect (Richardson)

Many extensions of Richardson's Theorem have have been found. An easy one is:

\author{
†hgaleana@matem.unam.mx \\ ¥guevara@matem.unam.mx \\ 1365-8050 @ 2005 Discrete Mathematics and Theoretical Computer Science (DMTCS), Nancy, France
}


Proposition 1 Let $D$ a digraph such that every cycle of odd length is symmetrical. Then $D$ is kernel perfect.

Others theorems have been found, in particular the following:

1. If every cycle of odd length $\left(x_{1}, x_{2}, \ldots x_{2 k+1}, x_{1}\right)$ has two pseudodiagonals of the type $\left(x_{i}, x_{i+2}\right)$, $\left(x_{i+1}, x_{i+3}\right)$ then the digraph is kernel perfect. (3)

2. If every cycle of odd length has at least two symmetrical arcs, then the digraph is kernel perfect.

A directed cycle of length 3 will be called a triangle and a forbidden triangle is a triangle with at most one symmetrical arc. $M$-oriented digraphs have no forbidden triangles. The covering number of a digraph $D$, denoted by $\theta(D)$ is the minimum number of complete subdigraphs of $D$ that partition $V(D)$.

The following are sufficient conditions for a $M$-oriented digraphs with $\theta(D) \leq 3$ is kernel perfect:

- If each directed cycle $\mathcal{C}$ of length 5 contained in $D$ satisfies at least one of the following properties: (a) $\mathcal{C}$ has two diagonals, (b) $\mathcal{C}$ has three symmetrical arcs.

- If every directed cycle of length 5 has three symmetrical arcs.

- If every directed cycle of length 5 has a symmetrical diagonal.

- If every directed cycle of length 5 has two diagonals.

A semikernel $S$ of $D$ is an independent set of vertices such that for every $z \in V(D)-S$ for which there exists an arc from a vertex in $S$ to $z$, there also exists an $\operatorname{arc}$ from $z$ to a vertex in $S$. Notice that a kernel $N$ of $D$ is a semikernel of $D$. A digraph $D$ is kernel perfect if every non-empty induced subdigraph of $D$ has a kernel. We say that $D$ is a critical kernel imperfect digraph if $D$ does not have a kernel but each proper induced subdigraph of $D$ does have at least one .

In (9), Neumann-Lara introduced the concept of a semikernel and, considering the kernel perfect digraphs, obtained sufficient conditions for the existence of a kernel in a digraph in terms of semikernels.

Teorema 2 (9) Let $D$ be a digraph. If every induced subdigraph of $D$ has a non-empty semikernel then $D$ is kernel perfect.

This result provides another equivalent definition of a kernel perfect digraph: a digraph is kernel perfect if every non-empty induced subdigraph has a non-empty semikernel.

Theorem 2 allows us to prove in a simpler way Richardson's Theorem (7), which originally had a long and complicated proof: any digraph which does not contain directed cycles of odd length has a kernel; its enough to prove that every bipartite digraph has a semikernel. Theorem 2 also provides tools to give some general sufficient conditions for a digraph to be a kernel perfect digraph and some structural properties on critical kernel imperfect digraphs. Therefore, the concept of a semikernel has been very important in the development of Kernel Theory.

In (5), Galeana-Sánchez introduced the following concept: let $F$ be a set of arcs of $D$. A set $S \subseteq V(D)$ is called a semikernel of $D$ modulo $F$ if $S$ is an independent set such that for every $z \in V(D)-S$ for which there exists an arc from a vertex in $S$ to $z$ of $D-F$, there also exists an $z S$-arc in $D$. We can observe that a semikernel $S$ is a semikernel modulo $F$, (for some $F$ ). 
A digraph $D$ will be called asymmetrically transitive whenever $u v, v w \in A \operatorname{sym}(D)$ implies $u w \in$ $\operatorname{Asym}(D)$, where $A \operatorname{sym}(D)$ is the spanning subdigraph of $D$ whose $\operatorname{arcs}$ are asymmetrical arcs of $D$.

In this work the concept of semikernel modulo $F$ is used to obtain new sufficient conditions for the existence of kernels in digraphs; this results are more general than those obtained by using the concept of semikernel and also apply for infinite digraphs.

An infinite sequence $\left(x_{1}, x_{2}, \ldots\right)$ of distinct vertices of $D_{1}$, such that $x_{i} x_{i+1} \in A\left(D_{1}\right)$ for each $i$ is called infinite outward path.

Teorema 3 Let $D$ be a (possibly infinite) digraph. Let $D_{1}$ be an asymetrically transitive subdigraph of $D$ without infinite outward path, such that every induced subdigraph of $D$ has a non-empty semikernel modulo $A\left(D_{1}\right)$. If $D$ has no induced subdigraph isomorphic to a member of a special family of 14 digraphs, then $D$ is a kernel perfect digraph.

We will provide an equivalent definition of a kernel perfect digraph for a class of digraphs; If $D$ satisfy:

- There exists $D_{1} \subset D$ such that, there is a partial order, $\leq$, in the set of non-empty semikernels of $D$ modulo $A\left(D_{1}\right)$, with a maximal element.

- If $S$ is a non-empty semikernel of $D$ modulo $A\left(D_{1}\right)$, such that $B_{S}=\{v \in D-S \mid \nexists v S-$ arc in $D\} \neq \emptyset$ and, if $S^{\prime}$ is a non-empty semikernel of $D\left[B_{S}\right]$ modulo $A\left(D_{1}\right)$, then $T_{S} \cup S^{\prime}$ is nonempty semikernel of $D$ modulo $A\left(D_{1}\right)$ and $T_{S} \cup S^{\prime}>S$, where $T_{S}=\left\{v \in S \mid \nexists v S^{\prime}-\operatorname{arc}\right.$ in $\left.D_{1}\right\}$.

- If $S_{0}$ is maximal with respect to $\leq$, then $S \subset S_{0} \cup\left\{x \in V(D) \mid \exists x S_{0}-\operatorname{arc}\right.$ in $\left.D\right\}$, for each $S<S_{0}$

we say that $D$ holds the property $P\left(\alpha_{D_{1}}, \leq\right)$. We say that $D$ satisfy hereditarily $P\left(\alpha_{D_{1}}, \leq\right)$ if $D$ holds the property $P\left(\alpha_{D_{1}}, \leq\right)$ and every $H \subset^{*} D$ holds $P\left(\alpha_{D_{1}[V(H)]}, \leq\right)$, with $\leq$ restricted to $\alpha_{D_{1}[V(H)]}$. Note that the independent sets of $H$ are also independent in $D$.

Teorema 4 Let $D$ be a digraph that satisfy hereditarilly $P\left(\alpha_{D_{1}}, \leq\right)$. D is kernel perfect if every nonempty induced subdigraph has a non-empty semikernel modulo $A\left(D_{1}\right)$.

Notice that Theorem 3 implies Theorem 2, if we have that $D_{1}$ is $\operatorname{Sym}(D)$ (the spanning subdigraph of $D$ whose arcs are symmetrical arcs of $D$ ). As a consequence of Theorem 3 , we obtain a generalization of the following result due to B. Sands, N. Sauer and R. Woodrow (8): Let D be a digraph whose arcs are colored with two colors. If D contains no monochromatic infinite outward path, then there exists a set $S$ of vertices of $D$ such that no two vertices of $S$ are connected by a monochromatic directed path and for every vertex not in $S$ there is a monochromatic directed path from $x$ to a vertex in $S$.

In (6), Galeana-Sánchez and V. Neumann-Lara, using the notions of semikernels, gave sufficient conditions for a digraph to be a kernel perfect digraph. Those conditions generalized those studied by, e.g. Duchet (2). As a example, we have:

Teorema 5 If every directed cycle $C$ of odd length in $D$ has two pseudodiagonals with consecutive terminal endpoints then $D$ is kernel perfect.

Galeana-Sánchez and Neumann-Lara also gave some structural properties of critical kernel imperfect digraphs. In particular they proved that every vertex (resp. arc) in a critical kernel imperfect digraph $D$, is contained in an odd directed cycle containing some "special pseudodiagonals". 
In this work, we generalize the results of Galeana-Sánchez and Neumann-Lara, using the notions of semikernels modulo $A\left(D_{1}\right)$, where $D_{1} \subset D$ and asking for $D$ to hold the property $P\left(\alpha_{D_{1}}, \leq\right)$, (the results of them are obtained if $D_{1}=\operatorname{Sym}(D)$ ).

The following theorems let us know some structures of the critical kernel imperfect digraphs:

We say that a cycle $C=\left(u_{0}, u_{1}, \ldots u_{n}\right)$ in $D$ alternate arcs, (resp. vertex), in $A \subset A(D)$, (resp. $B \subset V(D))$, if $u_{0} u_{1}, u_{2} u_{3}, \ldots$ in $A$, (resp. $\left.u_{0}, u_{2}, \ldots \in B\right)$.

Teorema 6 Every arc in a critical kernel imperfect digraph $D$ (possibly infinite) holding $P\left(\alpha_{D_{1}}, \leq\right)$ is contained in an odd directed cycle that alternate arcs in $A(D)-A\left(D_{1}\right)$ not containing special pseudodiagonals.

Remark: Up to now, it is not known if an infinite critical kernel imperfect digraph exists.

Teorema 7 Every vertex in a critical kernel imperfect digraph $D$ (possibly infinite), holding $P\left(\alpha_{D_{1}}, \leq\right)$, which is not a directed cycle of odd length, belongs to at least $\Delta_{D}(u)+1$ directed cycle of odd length that alternate arcs in $A(D)-A\left(D_{1}\right) .\left(\Delta_{D}(u)=\max \left\{\left|\Gamma^{-}(u)\right|,\left|\Gamma^{+}(u)\right|\right\}\right)$.

In particular, we provide sufficient conditions, as in the following theorems, to assure when a digraph is kernel perfect:

Teorema 8 Any finite digraph holding $P\left(\alpha_{D_{1}}, \leq\right)$ in which every odd directed cycle that alternate arcs in $A(D)-A\left(D_{1}\right)$, has two pseudodiagonals with consecutive terminal endpoints, is kernel perfect.

Denote by $\mathcal{V}_{D_{1}}$, (resp. $\mathcal{F}_{D_{1}}$ ), the set of vertices (resp. arcs) of $D$ which do not belong to a directed cycle of odd length that alternate arcs in $A(D)-A\left(D_{1}\right)$.

Teorema $9 D$ is kernel perfect digraph iff $D-\mathcal{V}_{D_{1}}$, (resp. every induced subdigraph $H$ of $D$ such that $\left.A(H) \cap \mathcal{F}_{D_{1}}=\emptyset\right)$, is a kernel perfect digraph.

\section{References}

[1] C. Berge, Graphs, North-Holland Mathematical Library, Vol. 6 (North-Holland, Amsterdam,1985), Chapter 14.

[2] P. Duchet, Representation; noyaux en theorie des graphes et hypergraphes, Thése, Paris (1979).

[3] P. Duchet, H. Meyniel, Une généralisation du théorème de Richardson noyaux dans le graphes orientés, Discrete Math. 43 (1983), 21-27.

[4] H. Galeana-Sánchez, Kernels in digraphs with covering number at most 3, Discrete Math., 259 (2002), no. 1-3, 121-135.

[5] H. Galeana-Sánchez, Semikernels modulo F and kernels in digraphs, Discrete Math., 218 (2000), 61-71.

[6] H. Galeana-Sánchez, V. Neumann-Lara, On kernels and semikernels of digraphs, Discrete Math., 48 (1984), $67-76$.

[7] M. Richardson, On weakly ordered systems, Bull. Amer. Math. Soc., 52 (1946), pag. 113. 
[8] B. Sands, N. Sauer and R. Woodrow, On monochromatic paths in edge-coloured digraphs, J. Combin. Theory, Ser. B 33 (1982), 271-275.

[9] V. Neumann-Lara, Seminúcleos de una digráfica, An Inst. Mat. Univ. Nac. Autónoma México, II (1971), 55-62.

[10] J. Von Neumann and O. Morgenstern, Theory of Games and Economic Behavior, Princeton University Press, Princeton, (1944). 
